\title{
动态热机械分析仪(DMA)在铁电压电材料研究中的应用
}

\author{
陈云, 王旭升, 李艳霞, 姚喜 \\ (同济大学 材料科学与工程学院, 上海 201804)
}

摘 要: 动态热机械分析仪(DMA)具有高灵敏度、卓越制冷技术、自由旋转测试头、多种形变模式和连续频率温度 扫描模式等优点, 能表征材料在交变应力(或应变)作用下的应变(或应力)的响应、蠕变、应力松弛和热机械性能等, 广泛应用于塑料、热固性材料、复合材料、高弹性体、涂层材料、金属和陶瓷等材料的研究和评估。本文简要介 绍了 DMA 进行动态力学行为分析的基本原理和方法, DMA 在铁电相变、低频弛豫特性和铁电瘦劳研究方面的应 用, 以及 DMA 在铁电/聚合物复合阻尼材料研究中的应用。在对 PZT 陶瓷和单晶、 $\mathrm{BaTiO}_{3}$ 陶瓷等常用材料的铁电 弛豫特性分析中, DMA 表现出比介电表征更为敏感的特性。现在 DMA 已成为研究铁电材料的重要工具之一。

关 键 词: 动态热机械分析仪; 铁电材料; 低频弛豫; 铁电疲劳; 阻尼; 综述 中图分类号: TQ174 文献标识码: A

\section{Dynamic Mechanical Analysis in the Investigation on Ferroelectrics}

\author{
CHEN Yun, WANG Xusheng, LI Yanxia, YAO Xi
}

(School of Materials Science and Engineering, Tongji University, Shanghai 201804, China)

\begin{abstract}
Dynamic mechanical analysis (DMA) has the advantage of high sensitivity, excellent cooling system, flexible rotation testing part, multiple deformation mode, and continuous frequency and temperature scanning mode. DMA is able to characterize the strain response under alternating stress, creep, stress relaxation, and thermomechanical properties, which has application in the investigation of plastic, thermoset, composite, high elastomer, coating, alloy and ceramic. This paper briefly introduced the fundamental and method about DMA, the application of DMA in the investigation of ferroelectric-paraelectric phase transformation, low frequency relaxation, ferroelectric fatigue, and ferroelectric composite damping. In the measurement of relaxation behavior of PZT ceramics and single crystals, and $\mathrm{BaTiO}_{3}$ ceramics, DMA tended to be more sensitive than dielectric characterization especially in the low frequency range. DMA has been one of the critical instruments for ferroelecric investigation.
\end{abstract}

Key words: dynamic mechanical analysis; ferroelectrics; low frequency relaxation; ferroelectric fatigue; damping; review

由于智能元件的蓬勃发展, 在电场、温度场和 磁场等外界刺激下能产生大机械应变的材料得到了 广泛的关注 ${ }^{[1-4]}$ 。高精度和快速的反应能力、高应变 储能密度、持久和可靠的服役寿命, 都是该类应变
材料的研究目标。相比形状记忆合金和磁致伸缩材 料, 铁电压电材料由于其高频反应和致动能力, 得 到广泛研究和应用 ${ }^{[5-7]}$, 大量应用于通信、传感器和 致动器、热成像和红外探测系统 ${ }^{[8-10]}$ 。其中, 弛豫型

收稿日期：2019-09-25; 收到修改稿日期：2019-11-12

基金项目：国家自然科学基金(51572195) National Natural Science Foundation of China (51572195)

作者简介: 陈 云(1990-), 女, 博士研究生. E-mail: xiaoyun369@126.com CHEN Yun(1990-), female, PhD candidate. E-mail: xiaoyun369@126.com

通讯作者: 王旭升，教授. E-mail: xs-wang@tongji.edu.cn WANG Xusheng, professor. E-mail: xs-wang@tongji.edu.cn 
铁电体具有巨大的机电耦合能力, 更是革新了整个 传感器和超声领域。Cross ${ }^{[11]}$ 最早定义了弛豫铁电体 的三大特征, 最大介电值对应的温度具有频率色 散、铁电相变宽泛弥散以及相变后高温段仍具有较 大剩余极化。

对于铁电压电材料, 除了保证本身的电学特 性外, 还必须确保服役期间其力学性能有效。传 统的力学测试方法多在静态下进行, 然而在实际 应用中铁电压电材料常受到动态交变载荷的作用, 如铁电材料在太空望远镜中应用 ${ }^{[12]}$ 、生态压电膜 在扬声器中的应用 ${ }^{[13]}$ 以及铁电复合材料在减震阻 尼方面的应用等 ${ }^{[14-16]}$ 。材料的动态力学行为, 指 材料在交变应力作用下的应变响应或交变应变作 用下的应力响应。因此, 相比常规的静态力学测 试, 测试动态力学行为更符合材料服役时的实际 情况。1909 年, Poynting ${ }^{[17]}$ 最早利用振荡实验研究 材料的动态力学性能, 随后在测试仪器方面历经 了流变振动仪、扭摆、扭辫分析、振簧以及动态 热机械分析仪 (DMA)。相比早年的测试仪器, DMA 具有更宽的频域和温域测试范围, 并实现了 频率无级连续调节, 能测试的材料参数包括: 储 能模量、损耗模量、损耗因子、玻璃化转变温度、 固化剂体系的软化温度、特征频率和特征松弛时 间等 ${ }^{[18]}$ 。

DMA 能模拟材料实际服役情况, 在不破坏样 品结构的条件下, 快捷准确地获得材料内部分子运 动转变的重要信息, 能定性、定量表征材料的热性 能、物理性能、机械性能及稳定性, 对于研究铁电 材料有着重要的指导意义。本文将简要介绍 DMA 测试的原理和方法、在铁电相变、低频弛豫特性和 铁电疲劳方面的研究应用, 以及 DMA 在铁电/聚合 物复合阻尼材料中的研究应用。

\section{DMA 的原理和方法}

DMA 测试的基本原理是将一个交变载荷施加 在样品上, 记录样品产生的响应, 输入样品的尺寸, 同时通过计算输出材料的动态性能参数 ${ }^{[19]}$ 。

若对材料施加正弦应力激励:

$$
\sigma(t)=\sigma_{0} \sin \omega t
$$

$\sigma$ 为即时应力, $\sigma_{0}$ 为最大应力, $\omega$ 为振荡频率, $t$ 为 时间。

对于理想弹性材料, 应变对应力的响应是瞬时 的, 故其为与应力同相位的正弦函数:

$$
\varepsilon(t)=\varepsilon_{0} \sin \omega t
$$

$\varepsilon$ 为即时应变, $\varepsilon_{0}$ 为最大应变。

对于理想的粘性材料, 应变则落后于应力 $90^{\circ}$ :

$$
\varepsilon(t)=\varepsilon_{0} \sin (\omega t-\pi / 2)
$$

对于粘弹性材料, 应变将滞后于应力一个相位 角 $\delta(0<\delta<\pi / 2)$ :

$$
\begin{gathered}
\varepsilon(t)=\varepsilon_{0} \sin (\omega t-\delta) \\
\varepsilon(t)=\varepsilon_{0}(\cos \delta \sin \omega t-\sin \delta \cos \omega t)
\end{gathered}
$$

由式(5)可以看出, 对于粘弹性材料, 应变响应 包含两部分, 一部分与应力同相位体现材料的弹性, 另一部分比应力落后 $90^{\circ}$ 体现材料的粘性。不同材 料应变落后于应力的相位差如图 1 所示。

材料的模量定义为应力和应变之比, 由于粘弹 性材料的应力与应变之间存在一个相位差, 因而模 量为复数。

$$
\begin{gathered}
\sigma=\sigma_{0} e^{\mathrm{i} \omega t} \\
\varepsilon(t)=\varepsilon_{0} e^{-\mathrm{i}(\omega t+\delta)} \\
E^{*}=\frac{\sigma(t)}{\varepsilon(t)}=\frac{\sigma_{0}}{\varepsilon_{0}} e^{\mathrm{i} \delta}=\frac{\sigma_{0}}{\varepsilon_{0}}(\cos \delta+\mathrm{i} \sin \delta)
\end{gathered}
$$

也即:

$$
E^{*}=\left|E^{*}\right|(\cos \delta+\mathrm{i} \sin \delta)=E^{\prime}+\mathrm{i} E^{\prime \prime}
$$

其中:

$$
\begin{gathered}
E^{\prime}=\frac{\sigma_{0}}{\varepsilon_{0}} \cos \delta \\
E^{\prime \prime}=\frac{\sigma_{0}}{\varepsilon_{0}} \sin \delta \\
\tan \delta=\frac{E^{\prime \prime}}{E^{\prime}} \\
\left|E^{*}\right|=\sqrt{E^{\prime 2}+E^{\prime 2}}
\end{gathered}
$$

式中, 复数模量 $E^{*}$ 的实数部分 $E^{\prime}$ 为材料在形变过程 中由于弹性变形而储存的能量, 称为储能模量, 其 在动态作用下测得, 与静态力条件下所测得的弹性 模量不同; 虚数部分 $E^{\prime \prime}$ 表征材料在形变过程中由于 粘性形变而以热耗散的能量, 称为损耗模量; $\delta$ 为损 耗角, $\tan \delta$ 为损耗因子。

另外, 柔性系数 $s^{*}$ 与弹性模量 $E^{*}$ 的关系 ${ }^{[19]}$ 为

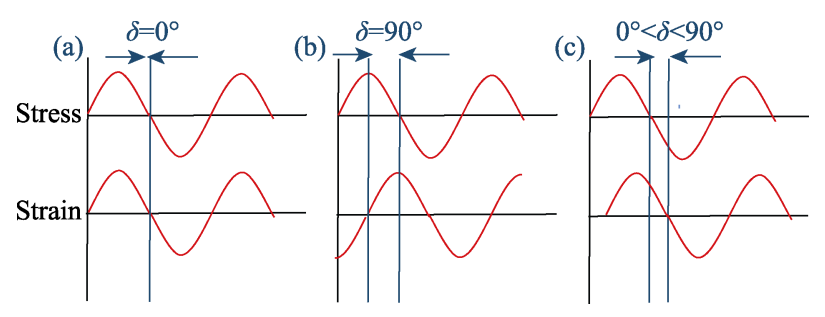

图 1 不同材料应变滞后于应力的相位差

Fig. 1 The lagging difference between strain and stress (a) Elastic material; (b) Viscous material; (c) Viscoelastic material 


$$
s^{*}=1 / E^{*}
$$

介电系数 $\varepsilon^{*}$ 和介电模量 $M^{*}$ 的关系 ${ }^{[20]}$ 为

$$
\varepsilon^{*}=1 / M^{*}
$$

从式(14 15)可以看出, 柔性系数与弹性模量、 介电系数和介电模量均具有倒数关系。通过压电方 程可知, 电学和力学参量具有如下关系 ${ }^{[21]}$ :

$$
\begin{gathered}
x=s^{E} X+d_{t} E \\
D=d X+\varepsilon^{X} E \\
d_{m i}=\varepsilon_{m n}^{X} g_{n i}=e_{m j} s_{j i}^{\mathrm{E}}
\end{gathered}
$$

其中, $x$ 为应变, $X$ 为应力, $D$ 为电位移, $E$ 为电场, $e 、 g$ 、 $d$ 为压电常数, $s$ 为柔性系数, $\varepsilon$ 为介电常数。由式 (16 18) 可知电学参量和力学参量能够相互表征, 虽 然各参量之间不具有明确的解析表达式，但具有很 明显的关联性。

同时，考虑到铁电材料在外场条件下的响应延 迟或者弛豫特征, 介电和柔性系数可以表达成复数 形式(式(19 20)), 因而, 理论上从电学和力学角度 均能表征铁电材料的弛豫特征。

$$
\begin{gathered}
\varepsilon^{*}=\varepsilon^{\prime}-\mathrm{j} \varepsilon^{\prime \prime} \\
s^{*}=s^{\prime}-\mathrm{j} s^{\prime \prime}
\end{gathered}
$$

DMA 的工作原理如图 2 所示 ${ }^{[22]}$ 。在设定温度 程序后(线性升温、降温、恒温或其组合), 对样品施 加单频或多频周期正弦波的动态振荡力, 样品将产
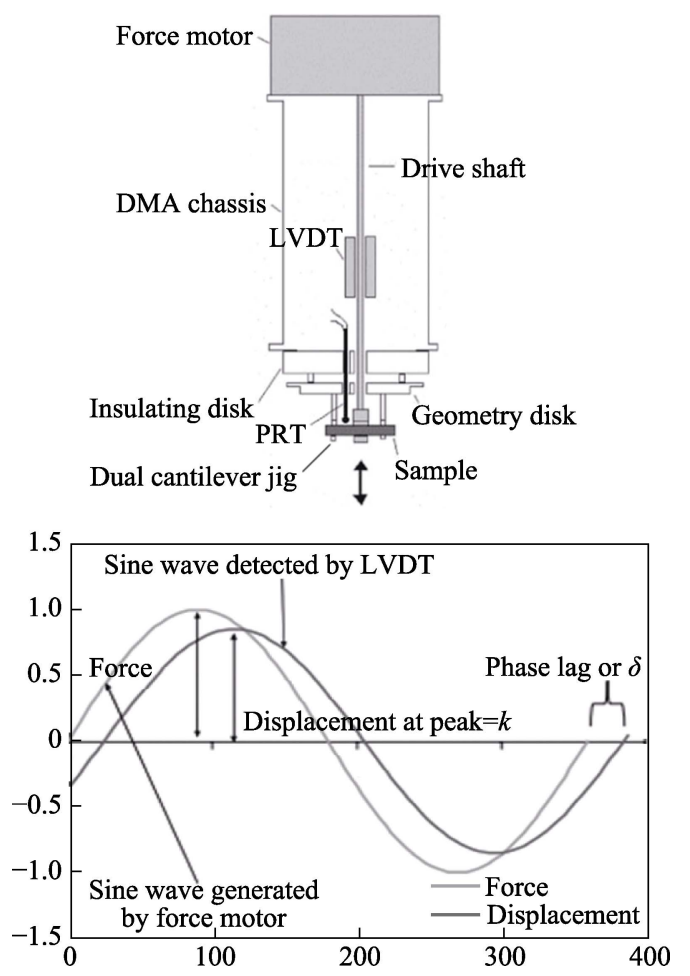

图 2 动态热机械分析仪(DMA)工作原理图 ${ }^{[22]}$

Fig. 2 Schematic diagram of dynamic mechanical analysis $(\mathrm{DMA})^{[22]}$
生响应的周期波动形变，该形变将经传感器并结合 相关的参数, 经数据处理得到储能模量 $E^{\prime}$ 、损耗模 量 $E^{\prime \prime}$ 和损耗因子 $\tan \delta$ 随温度变化的关系。

DMA 的测试模式主要包括单悬臂梁弯曲测试 法(Single cantilever)、双悬臂梁弯曲测试法(Dual cantilever)、三点弯曲测试法(3-point bending)、剪 切模式 (Shear)、拉伸模式 (Tension) 和压缩模式 (Compression)。

\section{DMA 在铁电材料相变研究中的应用}

铁电材料的重要特征是具有铁电相变, 对应的 温度为居里温度 $T_{\mathrm{C}}$, 在 $T_{\mathrm{C}}$ 之上材料将不具备铁电 性。研究材料铁电相变特点以及探测材料的居里温 度, 对确保铁电材料的正常服役具有十分重要的理 论和现实意义。从力学方面看, 材料的模量与其结 构和温度密切相关, 相变过程中由于应力和序参量 的耦合作用，材料结构和原子结合力改变，宏观上 表现为材料的模量软化, 因而模量对相变非常敏 感, 可以通过模量和温度的变化关系分析材料的相 变 ${ }^{[23]}$ 。同时, 弛豫、相界移动和新相形核均能产生 机械损耗峰。因此, 通过 DMA 记录样品的模量和 损耗随温度的变化, 可以分析被测样品的相变、缺 陷和弛豫等信息 ${ }^{[24-27]}$ 。

Zhang 等 ${ }^{[28]}$ 在研究局部应变各向异性对 $\mathrm{Ba}(\mathrm{Zr}, \mathrm{Ti}) \mathrm{O}_{3}-(\mathrm{Ba}, \mathrm{Ca}) \mathrm{TiO}_{3}(\mathrm{BZT}-\mathrm{BCT})$ 陶瓷高压电性能 的影响中, 利用 DMA 清楚地表征了 BZT-BCT 陶瓷 的各个相变, 如图 3 所示。从图 3(a)储能模量和温 度的关系以及(b)损耗和温度的对应关系可以看到, BZT-BCT 陶瓷的顺电相-四方相、四方相-正交相以 及正交相-斜方相相变点, 对应的相变温度分别约 在 360 $\left(T_{\mathrm{C}}\right) 、 304\left(T_{\mathrm{T}-\mathrm{O}}\right)$ 和 $273 \mathrm{~K}\left(T_{\mathrm{O}-\mathrm{R}}\right)$ 。通常在各个相 变点处, 模量软化并出现极小值, 因而利用 DMA 能清楚地表征铁电材料的各相变特点。

Silva 等 ${ }^{[29]}$ 研究了 $\mathrm{Bi}_{0.5} \mathrm{Na}_{0.5} \mathrm{TiO}_{3}-\mathrm{BaTiO}_{3}(\mathrm{BNT}-\mathrm{BT})$ 陶瓷体系在准同型相界(Morphotropic phase boundary, MPB)的相变行为, 并对比了介电温谱和力学参 数与温度的关系(图 4)。在图 4 的储能模量-温度曲 线上，一共出现 3 个异常峰，对应的温度分别为 $T_{1}$ 、 $T_{2}$ 和 $T_{3}$ 。其中, $T_{1} 、 T_{2}$ 分别对应斜方 - 四方相和四方 相-立方相转变, $T_{3}$ 对应四方铁电相-伪立方反铁电 相转变。Uddin 等 ${ }^{[30]}$ 也通过 DMA 研究了 BNT-BT 体系陶瓷的力学特征, 探测到了低温段的铁电一反 铁电相变, 以及较高温度段的反铁电一顺电相相变。

Puchberger 等 ${ }^{[31]}$ 利用 DMA 研究了 $\mathrm{PbZrO}_{3}$ 单晶 的电畴移动, 当施加的外力与单畴方向不同时, 对 单晶的相变具有不同影响, 其中当外力方向与单畴 

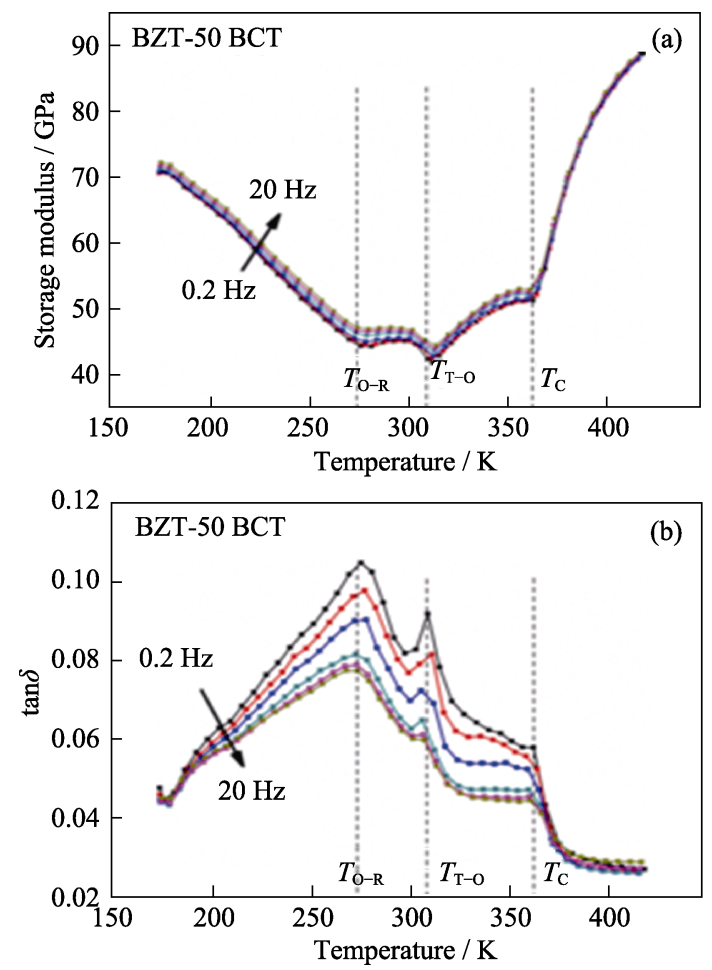

图 3 BZT-BCT 陶瓷的模量(a)和损耗因子 (b)与温度的关系 ${ }^{[28]}$ Fig. 3 Dependence of modulus (a) and loss factor (b) on temperature for BZT-BCT ceramic $^{[28]}$

垂直时，在反铁电相变处 $(510 \mathrm{~K})$ 会出现类似本征行 为的弹性异常峰(图 5)。由上文可知, 由于模量对相 变的高敏感度, DMA 可以作为检测铁电材料相变的 有效手段。

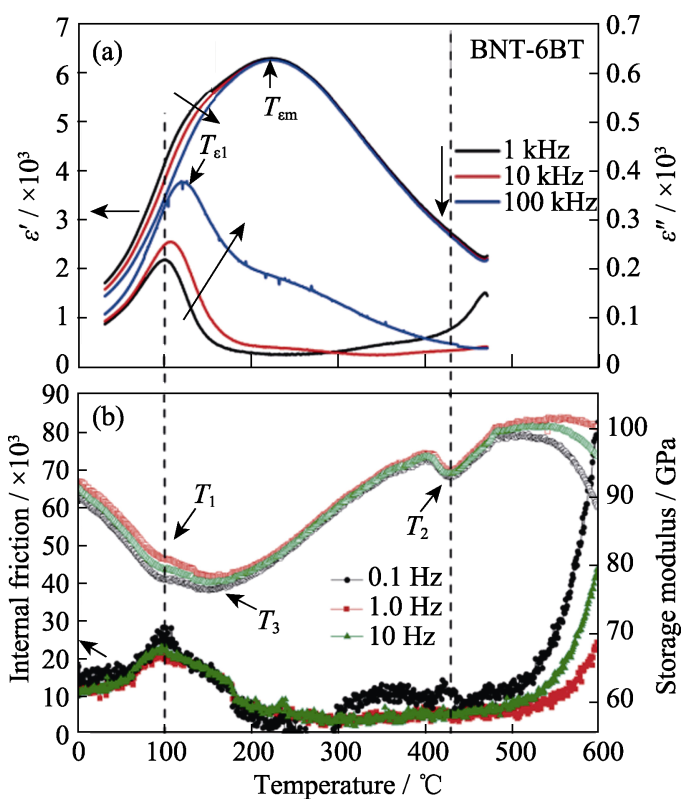

图 4 BNT-BT 陶瓷介电温谱(a)和力学图谱 $(b)^{[29]}$

Fig. 4 Dielectric spectra (a) and dynamic mechanical properties (b) of BNT-BT ceramic ${ }^{[29]}$

\section{DMA 在铁电材料弛豫特征研究方面 的应用}

通常，铁电体的弛豫特征通过介电常数随温度 变化的频率色散来表征, 并且频率越低弛豫特征越 明显，然而测量介电要求精确的夹持和屏蔽，难以 实现低频下的测量。DMA 则弥补了测试介电低频

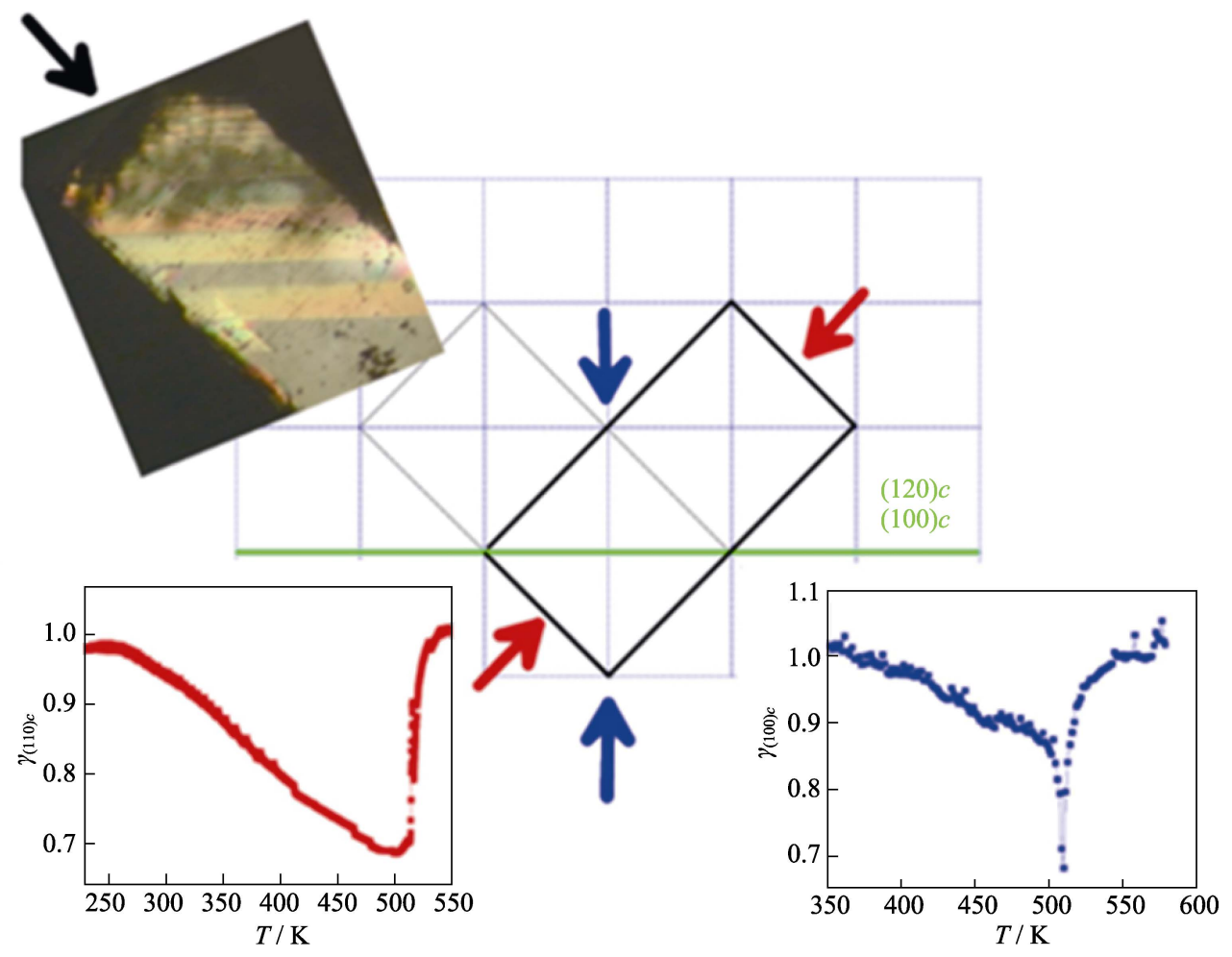

图 $5 \mathrm{PbZrO}_{3}$ 单晶对不同方向外力的弹性反应 ${ }^{[31]}$

Fig. 5 The elastic response to different external stress of $\mathrm{PbZrO}_{3}$ single crystal ${ }^{[31]}$ 
的不足, 甚至能实现超低频测试(低至 $0.001 \mathrm{~Hz}$ )。 Puchberger 等 ${ }^{[31}$ 研究了 $\mathrm{PbZrO}_{3}$ 单晶在不同方向的 动态力学性能, 其沿着 $[110]_{c}$ 方向的结果如图 6 所 示。模量的虚部对应机械损耗，不同频率下模量的 实部在 $500 \mathrm{~K}$ 左右急剧增加, 并且相应模量的虚部 有尖锐的损耗峰，该处对应 $\mathrm{PbZrO}_{3}$ 单晶的铁电-非 铁电相变。另外, 在模量的虚部图上还存在一个峰 位置随频率增加向高温方向移动的特征峰, 呈现出 明显的频率色散现象, 最低频率 $0.08 \mathrm{~Hz}$ 条件下, 峰 位置约在 $100 \mathrm{~K}$; 最高频率 $50 \mathrm{~Hz}$ 条件下, 峰位置约 在 $360 \mathrm{~K}$ 。该特征峰为典型的 Debye 弛豫峰, 并且 所得数据符合 Cole-Cole 弛豫方程。

DMA 不仅能表征铁电体的弛豫特征峰, 还能 研究弛豫的影响因素和机理。在对 $\mathrm{BaTiO}_{3}$ 陶瓷的动 态力学性能研究中, Cheng ${ }^{[32]}$ 观察到 $50{ }^{\circ} \mathrm{C}$ 左右出现 的弛豫峰与电畴壁相关。Jiménez 等 ${ }^{[33]}$ 在利用 DMA 研究 PCT 和 PST 陶瓷的低频动态力学行为时观察到 明显的低频弛豫峰，该弛豫峰与氧空位和电畴壁的 交互作用相关。在研究电畴运动对 PZT 陶瓷力学性 能的影响中, Bourim ${ }^{[34]}$ 观察到升温速率和加载大
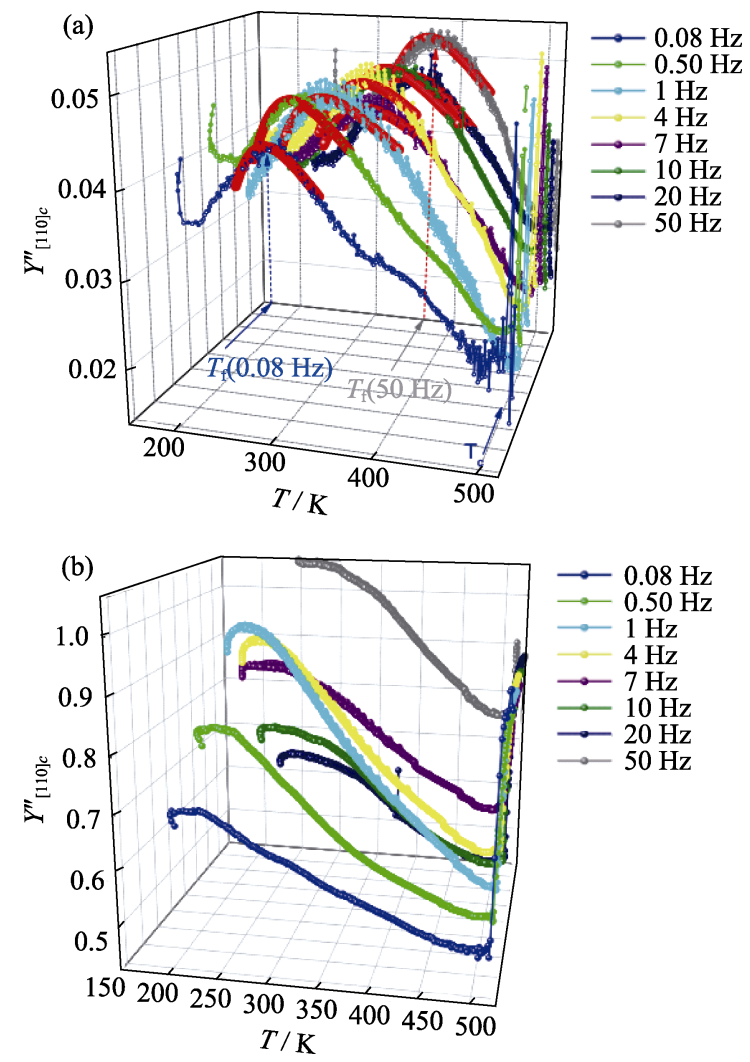

图 $6 \mathrm{PbZrO}_{3}$ 单晶沿着 $[110]_{c}$ 方向模量的实部 $(\mathrm{a})$ 和虚部(b)与 温度和频率的关系 ${ }^{[31]}$

Fig. 6 Dependence of modulus on temperature and frequency of $\mathrm{PbZrO}_{3}$ single crystal from $[110]_{c}$ direction

(a) Real part of modulus; (b) Imaginary part of modulus ${ }^{[31]}$
小对 R1 弛豫峰的峰值有影响，其中升温速率越慢 加载越大, R1 弛豫峰的峰值越大。Bourim ${ }^{[34]}$ 指出, R1 峰可能受如下机理控制: 电畴壁和点缺陷( $\mathrm{Ti} 、 \mathrm{Zr}$ 或者 $\mathrm{O}$ 空位)的相互作用; 居里温度附近电畴密度的 变化; 电畴受点缺陷和应力振幅效应的共同影响。

由于模量对材料结构变化的高敏感性以及 DMA 可实现超低频率测试, DMA 能更为精确地探 测铁电弛豫特征。在研究 PZT-4 陶瓷的低频弛豫特 征中发现力学表征和介电表征有差异，如图 $7^{[35]}$ 所 示。在 PZT-4 陶瓷的动态力学图谱图 7(a)中, 除去 相变峰 P1 外, 还存在一个随频率增加而向高温移动 的弛豫峰 P2。然而, 在介电图谱图 7(b)中, 只存在 一个相变峰, 并且在 $10 \mathrm{~Hz}$ 以下介电损耗异常增大, 不能完整表征。由此可见, 在较低频率范围, DMA 表征相比介电表征更有优势，能更完整地探测铁电 材料的弛豫特征。铁电陶瓷呈现出的机械和介电弛 豫特征都与陶瓷内部 $90^{\circ}$ 电畴壁的运动有关 ${ }^{[35-37]}$ 。 同时，由式(16 18)可知，力学参数与电学参数之间 能够相互转换和表征。因而，在外加应力场或电场 作用下，介电弛豫或者机械弛豫具有明显的关联性， 都是铁电体本身弛豫特征的不同表现形式。

对于具有扩散相变的弛豫铁电体，通常只能用 较复杂的手段去探测 $T_{\mathrm{f}}$, 如在变温条件下利用 TEM
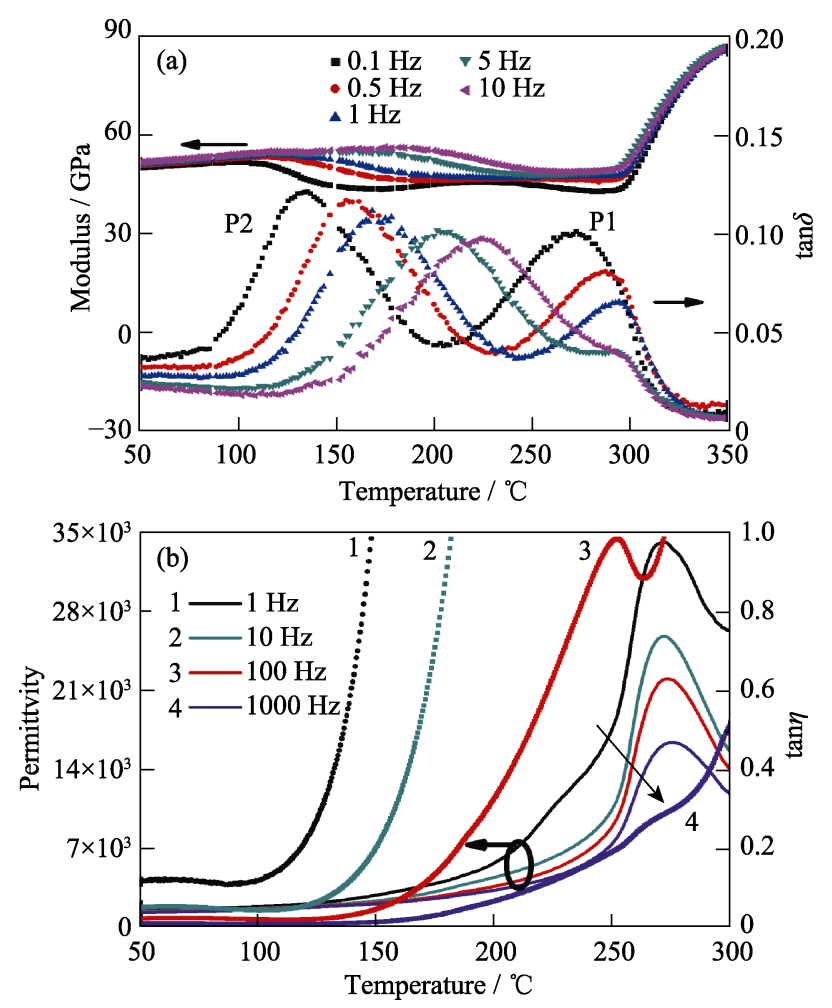

图 7 PZT-4 陶瓷的力学 $(a)$ 和介电图谱 $(b)^{[35]}$

Fig. 7 Dyanamic mechanical properties (a) and dielectric spectra (b) of PZT-4 ceramic $^{[35]}$ 
和 AFM 去直接观察极化微区。然而, 文献[38-40] 报道, 针对材料的滞弹性测量, 能够快捷有效地表 征出其 $T_{\mathrm{f}}$ 。图 8 为 $(\mathrm{K}, \mathrm{Na}) \mathrm{NbO}_{3}$ 基陶瓷的模量和损耗 随温度的变化曲线以及介电温谱 ${ }^{[39]}$ 。通常模量的突然 增大、损耗峰值处以及介电峰值处, 表征了铁电材料 的铁电一非铁电转变。在图 8 中, 模量突然增大的起始 温度低于介电峰值对应的温度, 而对于一般铁电体陶 瓷, 这两个温度一致。其原因在于 $(\mathrm{K}, \mathrm{Na}) \mathrm{NbO}_{3}$ 基陶瓷 是弛豫铁电体, 没有具体的铁电-非铁电相变点, 在 一段温域内动态极性微区发生扩散相变。由于滞弹 性表征比介电表征对结构变化更为敏感, 因而存在 表征温度差异。滞弹性表征对应扩散相变的起始点, 而介电表征温度对应平均值, 当一半的四方相已经 转变为立方相时, 开始出现介电峰值。因而, DMA 能够更为精确地描述扩散相变过程, 通过比较滞弹 性和介电表征, 能判定新材料是否具有扩散相变或 者弛豫特征。

综上, DMA 不仅能表征较低频率下介电方法无 法探测的弛豫特征, 研究铁电体的弛豫机理和影响 因素, 还能精确地表征弛豫铁电体的扩散相变特征。 因而, DMA 可以作为研究铁电体弛豫的有效手段。

\section{DMA 在铁电材料疲劳特性研究方面 的应用}

铁电存储器 FRAM 在数据采集与记录、参数设 置与存储、非易失性缓冲和储存扩展等方面有广泛

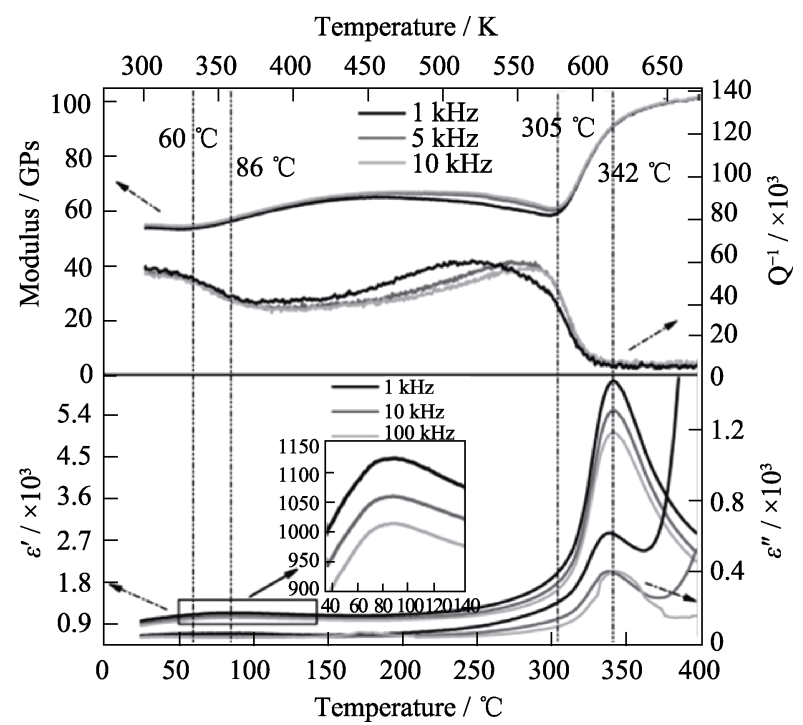

图 $8(\mathrm{~K}, \mathrm{Na}) \mathrm{NbO}_{3}$ 基陶瓷的模量和损耗与温度的变化关系以 及介电温谱 ${ }^{[39]}$

Fig. 8 Dielectric and dynamic mechanical properties of $(\mathrm{K}, \mathrm{Na})$ $\mathrm{NbO}_{3}$ based ceramic ${ }^{[39]}$
应用, 如电气仪表、GPS 定位系统、打印机、ATM 机和便携设备一体化存储器等。然而, 铁电薄膜及 储存器也存在可靠性问题, 如疲劳(Fatigue)、印记 失效 (Imprint failure) 和保持性能损失 (Retention loss)。在巨量的畴壁循环下, 铁电薄膜材料极易出 现电疲劳。典型特征为随着电场循环次数的增加, 微观可转动畴减少, 宏观剩余极化强度降低。疲劳 问题严重限制了铁电存储的商业化进程, 研究铁 电疲劳的起源及其影响因素具有重大的理论和实 际研究意义。

$\mathrm{Yu}$ 等 ${ }^{[41}$ 通过 DMA 研究了不同载荷下 PZT-5H 和 PZT-8 陶瓷模量的疲劳性能, 如图 9 所示。从 图 9(a)可知, 在经历 $7 \times 10^{7}$ 的载荷循环后, PZT- $5 \mathrm{H}$ 和 PZT-8 的模量均没有下降, 没有显示力学疲劳特征。 图 9(b)显示, 经过 $1 \times 10^{7}$ 次载荷循环后, PZT-5H 和 PZT-8 的压电系数 $d_{33}$ 分别下降了 $9 \%$ 和 $16 \%$, 经历 $7 \times 10^{7}$ 次载荷循环后，其 $d_{33}$ 分别下降了 $16 \%$ 和 $18 \%$ 。 该项研究对铁电致动器的疲劳特性研究和应用具有 指导意义。尽管模量变化不一定能精确反映疲劳程 度, 但通过 DMA 去检测材料的度劳性能, 还可以 通过其他参数表征, 如力-位移曲线或 S-N 曲线进 行表征和判断。

Xie 等 ${ }^{[42]}$ 利用 DMA 的三点弯曲模式研究了 BTWC 陶瓷的断裂机理和疲劳性能, BTWC 陶瓷在 $50 \mathrm{~Hz}$ 下的 $S-N$ 曲线如图 10(a)所示, 箭头表示达到
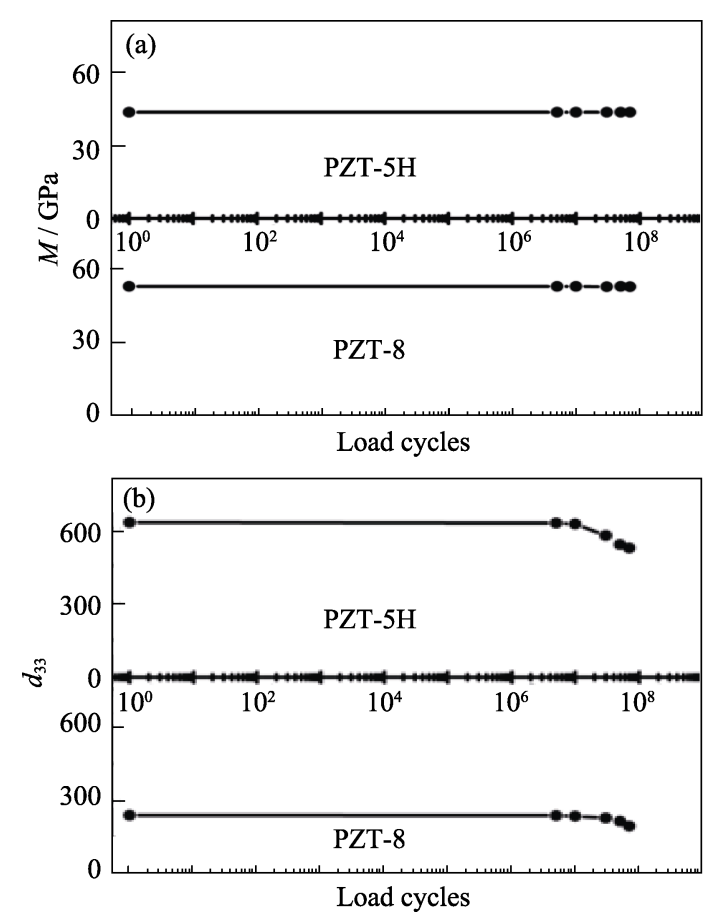

图 9 PZT-5H 和 PZT-8 陶瓷的疲劳性能 ${ }^{[41]}$

Fig. 9 Fatigue properties of PZT-5H and PZT- 8 ceramics $^{[41]}$ (a) Modulus vs circles; (b) $d_{33} v s$ circles 

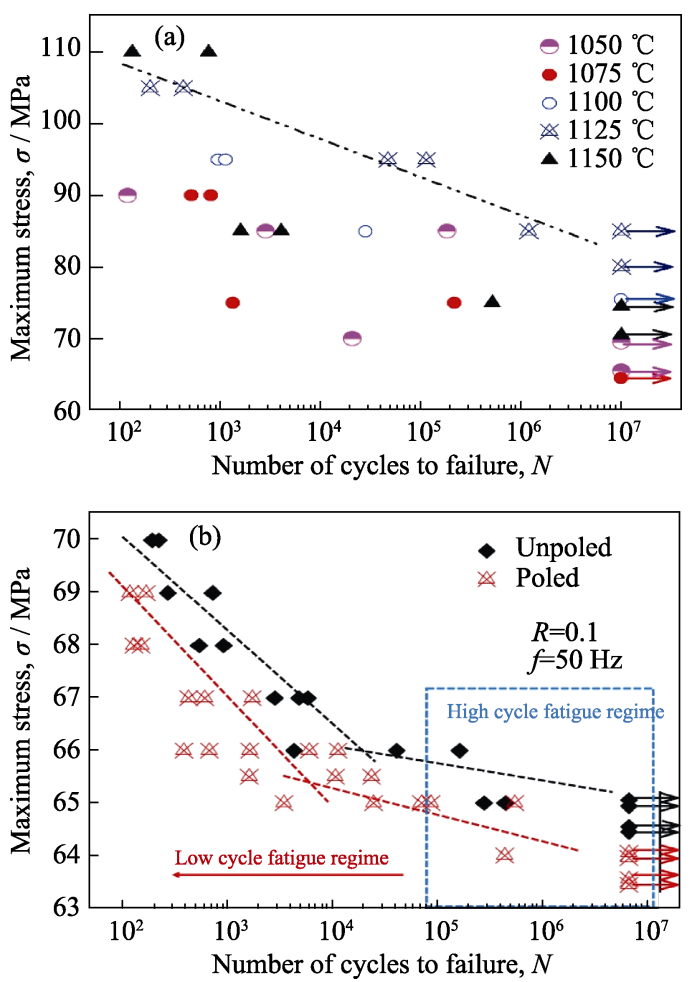

图 10 BTWC 陶瓷的 $S-N$ 曲线 ${ }^{[42-43]}$

Fig. 10 The $S-N$ curves of BTWC ceramics ${ }^{[42-43]}$

(a) BTWC ceramics sintered at different temperatures; (b) Unpoled and poled BTWC ceramics sintered at $1150{ }^{\circ} \mathrm{C}$

$1 \times 10^{7}$ 次循环后，陶瓷还没有发生断裂失效。结果表 明, BTWC 陶瓷的疲劳机理不仅和电畴结构有关,还 与陶瓷的孔洞和杂质有关。同时, Xie 等 ${ }^{[43]}$ 还利用 DMA 研究了极化对 BTWC 陶瓷疲劳性能的影响, 如图 10(b)所示。其结果表明，极化对陶瓷的疲劳 性能有负面影响，导致疲劳断裂向低周循环次数 移动。

目前，关于铁电疲劳的研究多集中于常温下的 电疲劳, 而力学疲劳及变温疲劳鲜有报道。在实际 应用中，铁电器件通常在电场、应力场和温度场共 同作用下工作。因而，过去大部分单独对电疲劳的 研究不能从根本上阐明疲劳损伤机理。DMA 能弥 补应力场和温度场下疲劳研究的不足, 有利于对铁 电疲劳的本质进行更深入的研究, 进一步从根本上 阐明铁电材料的疲劳机理。

\section{DMA 在铁电/聚合物复合阻尼材料 研究中的应用}

DMA 广泛应用于聚合物阻尼材料的研究, 用 以检测聚合物的玻璃化转变温度、弹性模量和阻尼 因子。近年来, 由于高速列车、飞机、潜艇、汽车 等高速交通工具减振降噪的需求, 对阻尼材料的需
求日益增加，各国都增加投入开发高性能阻尼材料， 其中压电复合阻尼材料显示出了巨大的优势和应用 前景。压电复合材料的阻尼性能综合了多种能量消 耗机制, 主要包括 ${ }^{[44-45]}$ : 基体高分子材料的粘弹性 行为产生的力学损耗, 将振动能转换为热能; 基体 材料和填充粒子的摩擦以及填充粒子间的相互摩擦 消耗的动能; 压电陶瓷粒子将振动机械能转为电能, 电能在一定的导电网络中转换为热能; 材料的介电 损耗消耗一部分能量。同时，由于高分子材料的高 粘滞性, 以及 DMA 能模拟各种振动条件, DMA 在 压电复合材料阻尼的机理和影响因素研究方面具有 广泛应用。

$\mathrm{Yu}$ 等 ${ }^{[46]}$ 利用 DMA 研究论证了 $\mathrm{BaTiO}_{3} / \mathrm{VGCF} /$ LDPE 复合材料的阻尼机理，尽管在复合材料中确 实存在压电效应，但只在特定条件下压电效应才能 发挥明显作用。在复合体系中, 压电效应主要受三 个因素控制：压电陶瓷颗粒和高分子基体的振动耦 合行为、压电颗粒的机电耦合因子大小、复合体系 的电导大小。Marra ${ }^{[47]}$ 在热塑性树脂 PVDF 和热固 性的环氧树脂中分别加入 $\mathrm{Ca}$ 改性的压电陶瓷 PT。 其结果表明, 加入压电陶瓷含量为 $52 \mathrm{vol} \%$ 时, PVDF 体系的储能模量从 $1.5 \mathrm{GPa}$ 提高到 $10 \mathrm{GPa}$, 损耗模 量从 $50 \mathrm{MPa}$ 提高到 $400 \mathrm{MPa}$ ，损耗因子 $\tan \delta$ 提高大 于 1 倍; 环氧树脂的储能模量从 $4 \mathrm{GPa}$ 提高到 $16 \mathrm{GPa}$, 损耗模量从 $20 \mathrm{MPa}$ 提高到 $280 \mathrm{MPa}, \tan \delta$ 提高大于 3 倍。由此可见, 通过 DMA 测试和比较材料体系的 模量和损耗因子，热塑性树脂相比热固性树脂具有 较低的玻璃化转变温度, 与压电颗粒复合后具有更 好的阻尼性能。

Zhang 等 ${ }^{[48]}$ 利用 DMA 研究了 0-3 型 PZT/PU 压 电复合物的动态力学性能, 发现在 PZT 颗粒含量不 多于 $30 \%$ 时，能在 PU 基体中均匀分布。复合体的 储能模量随着 PZT 颗粒的增加而升高, 机械损耗峰 向高温移动, 并且 PZT 能明显拓宽材料的阻尼作用 范围 $\left(70{ }^{\circ} \mathrm{C}\right)$, 但是损耗模量是随着 PZT 含量的增加 而下降的。

Hong 等 ${ }^{[49]}$ 利用 DMA 研究了 PZT 纤维/环氧树 脂和 $\mathrm{ZnOw} /$ 环氧树脂的阻尼性能，如图 11 所示。结 果表明, 两种复合材料的机械损耗均具有频率色散 特性, 机械损耗峰值随着频率增加而增加, 储能模 量随着频率增加而稍有上升, 因而在较高频率和较 大位移条件下，阻尼性能发挥更为明显。同时，1-3 型的 PZT/环氧树脂相比 0-3 型的 $\mathrm{ZnOw}$ /环氧树脂更 能发挥压电阻尼效应。 

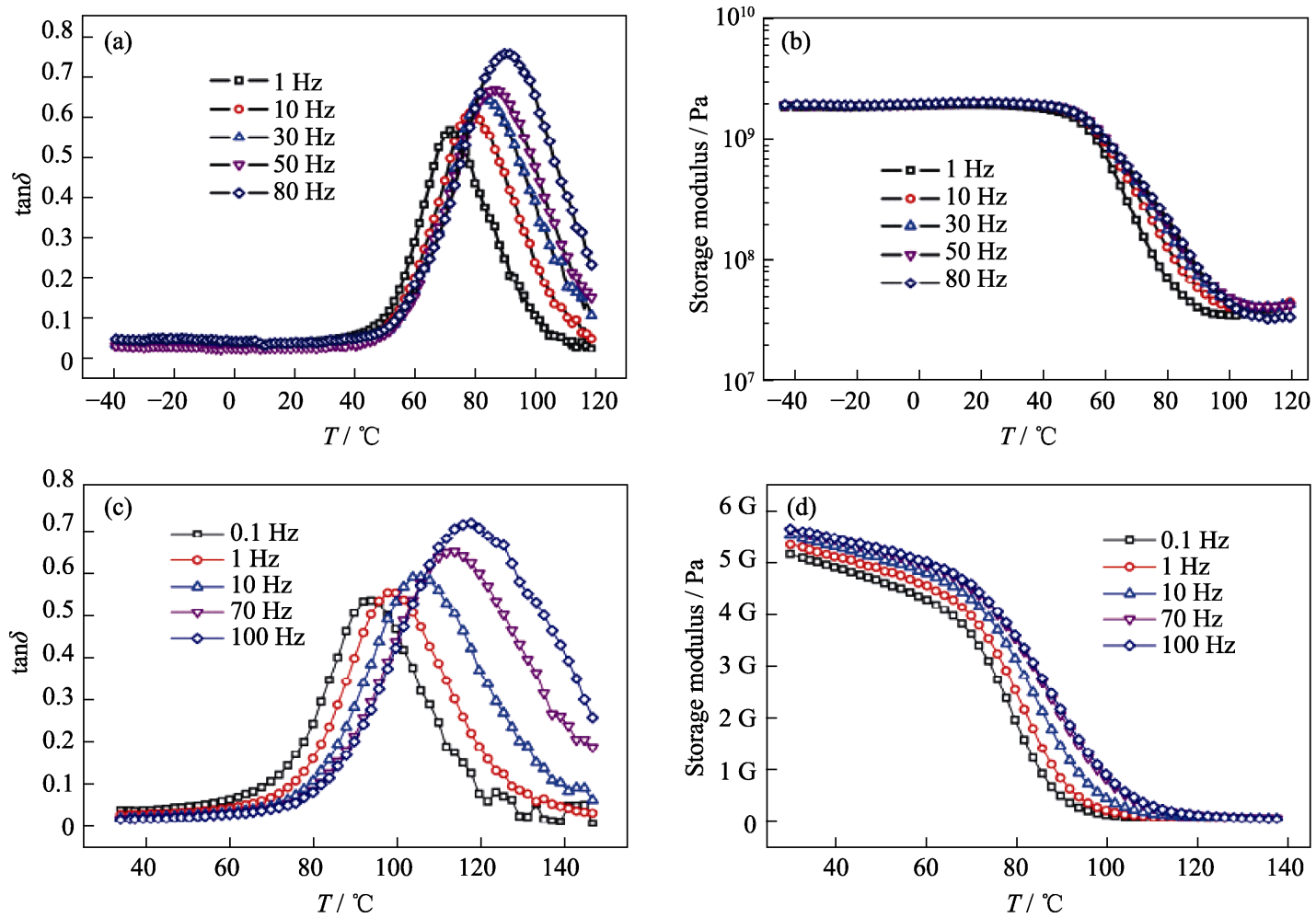

图 11 1-3 型 PZT/环氧树脂的动态力学性能 $(\mathrm{a} \sim \mathrm{b})$ 和 0-3 型 $\mathrm{ZnOw} /$ 环氧树脂动态力学性能 $(\mathrm{c} \sim \mathrm{d})^{[49]}$

Fig. 11 Dynamic mechanical properties of 1-3 type PZT/epoxy resin composite (a-b) and dynamic mechanical properties of 0-3 type $\mathrm{ZnOw} /$ epoxy resin composite (c-d $)^{[49]}$

Shi 等 ${ }^{[50]}$ 利用 DMA 研究了 0-3 型 PMN/CB/EP 压电复合材料的阻尼性能和导电体含量的关系。具 体结果如表 1 所示, 当碳黑 CB 含量为 $6 \mathrm{wt} \%$ 时, 复 合材料经由绝缘体转换为半导体, 并且机械损耗 因子 $\tan \delta_{\max }$ 、损耗区域面积 $(T A)$ 和阻尼作用温度 范围 $\Delta T$ 达到最大值。

武传贵 ${ }^{[51]}$ 利用 DMA 研究了 $0-3$ 型 PZT/环氧树 脂压电复合材料中 PZT 陶瓷颗粒粒径大小对阻尼性 能的影响。如图 12(a b)所示, 在玻璃态区掺有 1250 目 $(12 \mu \mathrm{m}) \mathrm{PZT}$ 颗粒样品的阻尼性能最佳, 且储能模 量最高。当颗粒过大, PZT 颗粒包含多个随机取向的

表 1 CB 含量和 0-3 型 PMN/CB/EP 压电 复合材料阻尼性能的关系 ${ }^{[50]}$

Table 1 The relationship between $C B$ content and the properties of 0-3 type $\mathrm{PMN} / \mathrm{CB} / \mathrm{EP}$ ferroelectric composite damping ${ }^{[50]}$

\begin{tabular}{crccc}
\hline $\mathrm{CB} / \mathrm{wt} \%$ & $T_{\mathrm{g}} /{ }^{\circ} \mathrm{C}$ & $\tan \delta_{\max }$ & \multicolumn{1}{c}{$T A$} & $\Delta T /{ }^{\circ} \mathrm{C}(\tan \delta>0.3)$ \\
\hline 0 & 85.86 & 0.405 & 18.21 & 20.28 \\
2 & 96.73 & 0.442 & 19.27 & 22.81 \\
4 & 100.26 & 0.429 & 18.73 & 20.86 \\
6 & 88.76 & 0.480 & 20.80 & 24.26 \\
8 & 93.40 & 0.439 & 19.64 & 21.23 \\
\hline
\end{tabular}

电畴，宏观上不呈现压电效应，只有在适当粒径范 围, 表现为单畴并发挥压电效应。此外, 其还利用 DMA 研究了不同电学边界条件对 1-3 型 PZT/环氧 树脂压电复合材料阻尼的影响。从图 12(c $\sim \mathrm{d})$ 可看 出, 当压电复合材料外接电阻时, 阻尼效果最为明 显, 并且测试频率越高, 损耗因子峰值越往低温方 向移动。

\section{6 总结与展望}

综上所述, DMA 已广泛用于铁电材料的研究, 包括对铁电相变的表征、对低频弛豫特性的表征和 研究、在铁电疲劳方面的研究以及在铁电/聚合物复 合阻尼材料中的研究应用。一方面, DMA 能与传统 的电学表征相配合, 弥补电学测试在低频方面的欠 缺不足, 更为全面地探测和理解铁电材料的结构和 性能。另一方面, 热分析联用技术已实现多领域交 叉应用，随着 DMA 在铁电材料研究的应用拓宽， 开发 DMA 与其他分析测试手段的联用技术是未来 的发展趋势, 如开发 DMA-SEM 在微视野中观测电 畴运动, DMA-AFM 同时探究电畴宏观与微观(原子) 力学作用变化。合理应用 DMA, 对于铁电材料的深 入研究和理解, 具有重要的理论和实际意义。 

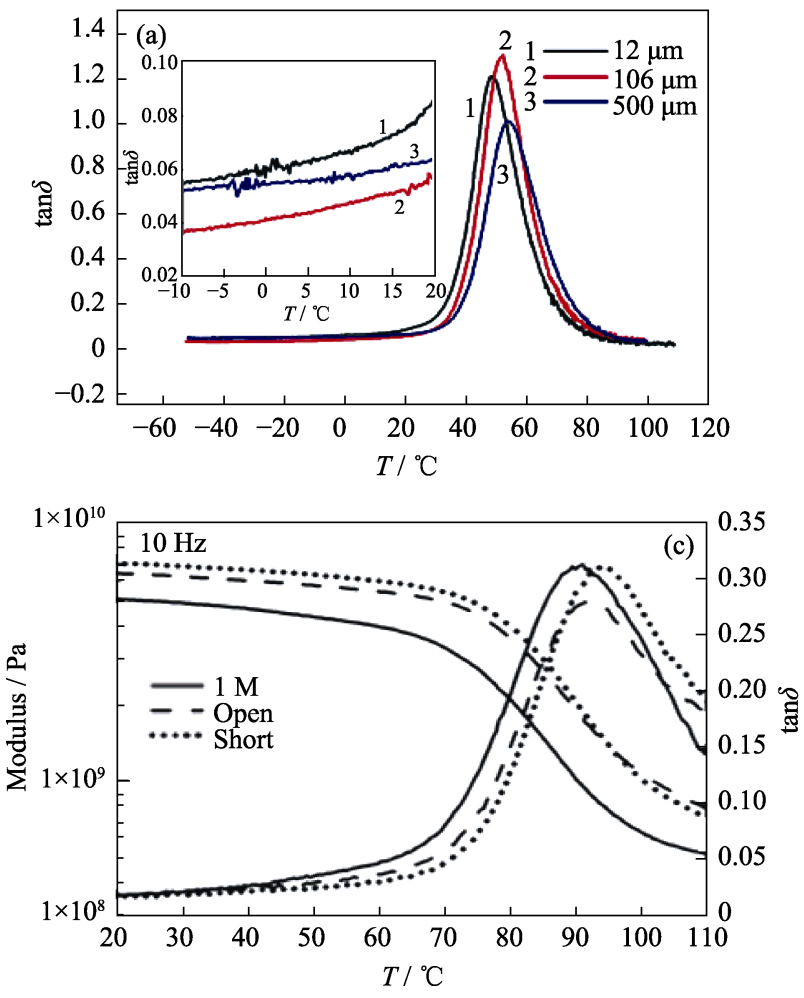
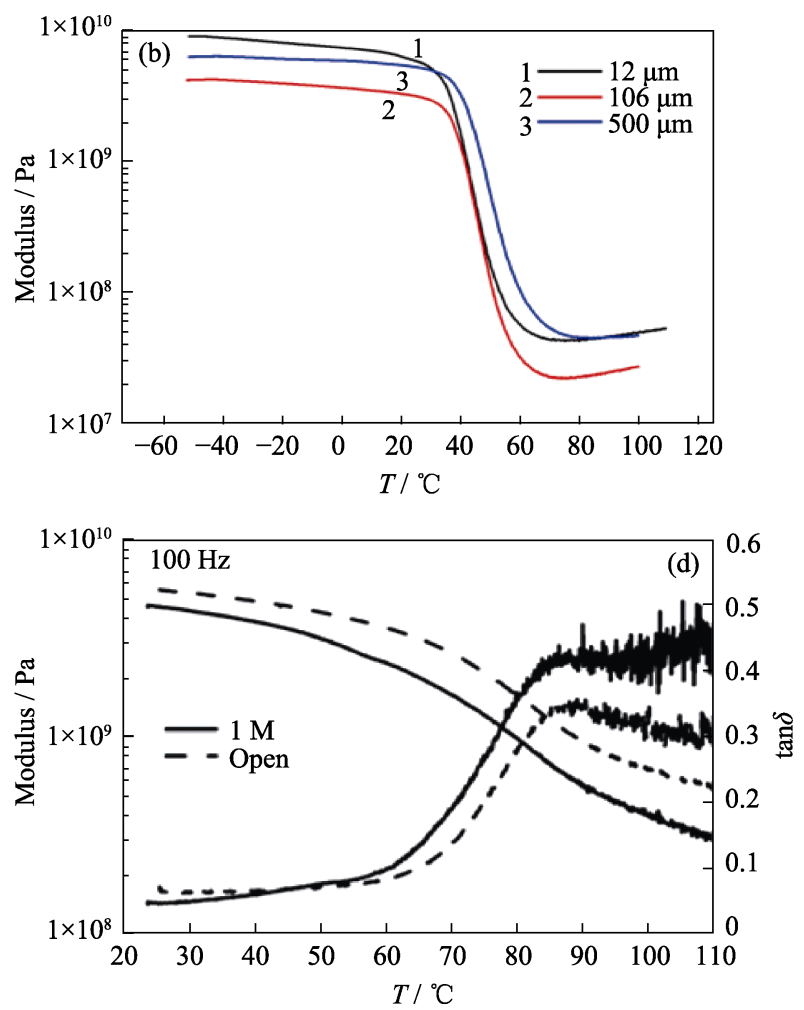

图 12 PZT 颗粒粒径和电学边界对压电复合材料的影响

(a)PZT 颗粒粒径对 0-3 型压电复合材料阻尼性能的影响; (b)PZT 颗粒粒径对 0-3 型压电复合材料储能模量的影响; (c-d)不同电学边界对 1-3 型 PZT 压电复合材料阻尼性能的影响 ${ }^{[51]}$

Fig. 12 Influence of grain size and electrical boundary on piezoelectric composite

(a) Influence of PZT grain size on the loss factor of 0-3 type piezoelectric composites; (b) Influence of PZT grain size on the modulus of 0-3 type piezoelectric composites; (c-d) Influence of different electrical boundary conditions on the dynamic mechanical properties of 1-3 type piezoelectric composites ${ }^{[51]}$

\section{参考文献:}

[1] DOMENJOUD M, BERTHELOT E, GALOPIN N, et al. Characterization of giant magnetostrictive materials under static stress: influence of loading boundary conditions. Smart Mater. Struct., 2019, 28: 095012.

[2] LIU N, ACOSTA M, WANG S, et al. Revealing the core-shell interactions of a giant strain relaxor ferroelectric $0.75 \mathrm{Bi}_{1 / 2} \mathrm{Na}_{1 / 2} \mathrm{TiO}_{3}$ 0.25: $\mathrm{SrTiO}_{3}$. Sci. Rep., 2016, 6: 36910.

[3] LIU X, TAN X, Giant strains in non-textured $\left(\mathrm{Bi}_{1 / 2} \mathrm{Na}_{1 / 2}\right) \mathrm{TiO}_{3}-$ based lead-free ceramics. Adv. Mater, 2016, 28(3): 574-578.

[4] LI T, LOU X, KE X, et al. Giant strain with low hysteresis in A-site-deficient $\left(\mathrm{Bi}_{0.5} \mathrm{Na}_{0.5}\right) \mathrm{TiO}_{3}$-based lead-free piezoceramics. Acta Mater, 2017, 128(15): 337-344.

[5] LESTER B T, BAXEVANIS T, CHEMISKY Y, et al. Review and perspectives: shape memory alloy composite systems. Acta Mech., 2015, 226(12): 3907-3960.

[6] NARITA F, FOX M. A review on piezoelectric, magnetostrictive, and magnetoelectric materials and device technologies for energy harvesting applications. Adv. Eng. Mater, 2018, 20: 1700743.

[7] WEBBER K G, VOEGLER M, KHANSUR N H, et al. Review of the mechanical and fracture behavior of perovskite lead-free ferroelectrics for actuator applications. Smart Mater. Struct., 2017, 26(6): 063001.

[8] SADEGHPOUR S, MEYERS S, KRUTH J P, et al. Single-element omnidirectional piezoelectric ultrasound transducer for under water communication. Proceedings, 2017, 1: 363.

[9] MA H K, LUO W F, LIN J Y. Development of a piezoelectric mi- cropump with novel separable design for medical applications. Sensor Actuat. A Phys., 2015, 236: 57-66.

[10] ZHOU M, LIANG R, ZHOU Z, et al. Potentiality of Bi and Mn co-doped lead-free $\mathrm{NaNbO}_{3}$ ceramics as a pyroelectric material for uncooled infrared thermal detectors. J. Eur. Ceram. Soc., 2019, 39(6): 2058-2063.

[11] CROSS L E. Relaxor ferroelectric. Ferroelectrics, 1987, 76(1): 241-267.

[12] STEEVES J B, GOLINVEAUX F S. Using the ferroelectric/ ferroelastic effect at cryogenic temperatures for set-and-hold actuation. Smart Mater. Struct., 2018, 27(6): 065024.

[13] KWEON S Y, LEE K, PARK Y, et al. Low-temperature sintering of $(1-x) \mathrm{Pb}\left(\mathrm{Zr}_{0.53} \mathrm{Ti}_{0.47}\right) \mathrm{O}_{3}-x \mathrm{BiYO}_{3}$ ceramics with nano-powder for piezo-speaker. Jpn. J. Appl. Phys., 2019, 58(5): 051008.

[14] MARAKAKIS K, TAIRIDIS G K, KOUTSIANITIS P. Shunt piezoelectric systems for noise and vibration control: a review. Fron. Built Environ., 2019, 5: 64.

[15] GRIPP J A B, RADE D A. Vibration and noise control using shunted piezoelectric transducers: a review. Mech. Syst. Signal PR., 2018, 112: 359-383.

[16] XU Z, CHEN Z, HUNAG X, et al. Recent advances in multidimensional vibration mitigation materials and devices. Fron. Mater., 2019, 6: 00143.

[17] POYNTING J H. On pressure perpendicular to the shear planes in finite pure shears, and on the lengthening of loaded wires when twisted. Proceedings of the Royal Society of London. Series A, 1909, 82(557): 546-559.

[18] GUO L M. The advanced dynamic mechanical thermal analysis 
(DMTA) and its applications. Modern Scientific Instrumentation, 1997, 4: 57-60.

[19] LI Z, SUN D, YAN B, et al. Fractional order model of viscoelastic suspension for crawler vehicle and its vibration suppression analysis. Transactions of the Chinese Society of Agricultural Engineering, 2015, 31(7): 72-79.

[20] 过梅丽. 高聚物与复合材料的动态力学热分析. 北京: 化学工 业出版社, 2002.

[21] 张良荣. 电介质物理. 西安: 西安交通大学出版社, 1991.

[22] MENARD H P. Dynamic Mechanical Analysis. Florida: CRC Press, 2008.

[23] YAN F, BAO P, WANG Y. Phase transition in relaxor ferroelectrics studied by mechanical measurements. Appl. Phys. Lett., 2003, 83(21): 4384-4386.

[24] CORDERO F. Hopping and clustering of oxygen vacancies in $\mathrm{SrTiO}_{3}$ by anelastic relaxation. Phys. Rev. B, 2007, 76(17): 172106.

[25] DIAZ J C C A, VENET M, CORDERO F, et al. Anelastic and optical properties of $\mathrm{Bi}_{0.5} \mathrm{Na}_{0.5} \mathrm{TiO}_{3}$ and $\left(\mathrm{Bi}_{0.5} \mathrm{Na}_{0.5}\right)_{0.94} \mathrm{Ba}_{0.06} \mathrm{TiO}_{3}$ leadfree ceramic systems doped with donor $\mathrm{Sm}^{3+}$. J. Alloy. Compd., 2018, 746(25): 648-652.

[26] ALGUERÓ M, JIMÉNEZ H, AMORÍN, et al. Low temperature phenomena in ferroic $\mathrm{BiMO}_{3}-\mathrm{PbTiO}_{3}(\mathrm{M}$ : Mn and Sc). Appl. Phys. Lett., 2011, 98(20): 202904.

[27] ZHANG D, YAO Y, FANG M, et al. Isothermal phase transition and the transition temperature limitation in the lead-free $(1-x)$ $\mathrm{Bi}_{0.5} \mathrm{Na}_{0.5} \mathrm{TiO}_{3}-x \mathrm{BaTiO}_{3}$ system. Acta Mater., 2016, 103: 746-753.

[28] ZHANG L, REN X, CARPENTER M A. Influence of local strain heterogeneity on high piezoelectricity in $0.5 \mathrm{Ba}\left(\mathrm{Zr}_{0.2} \mathrm{Ti}_{0.8}\right) \mathrm{O}_{3^{-}}$ 0.5( $\left(\mathrm{Ba}_{0.7} \mathrm{Ca}_{0.3}\right) \mathrm{TiO}_{3}$ ceramics. Phys. Rev. B, 2017, 95(5): 054116.

[29] SILVA P, DIAZ J, FLORÉNCIO, et al. Analysis of the phase transitions in BNT-BT lead-free ceramics around morphotropic phase boundary by mechanical and dielectric spectroscopies. Arch. Metall. Mater., 2016, 61(1): 17-20.

[30] UDDIN S, ZHENG G P, LQBAL Y, et al. Elastic softening near the phase transitions in $(1-x) \mathrm{Bi}_{1 / 2} \mathrm{Na}_{1 / 2} \mathrm{TiO}_{3}-x \mathrm{BaTiO}_{3}$ solid solutions. Mater. Res. Express, 2014, 1(4): 046102.

[31] PUCHBERGER S, SOPRUNYUK V, MAJCHROWSKI A, et al. Domain wall motion and precursor dynamics in $\mathrm{PbZrO}_{3}$. Phys. Rev. B, 2016, 94(21): 214101 .

[32] CHENG B, GABBAY M, FANTOZZI G. Anelastic relaxation associated with the motion of domain walls in barium titanate ceramics. J. Mater. Sci., 1996, 31(15): 4141-4147.

[33] JIMÉNEZ B, VICENTE J. Oxygen defects and low-frequency mechanical relaxation in $\mathrm{Pb}-\mathrm{Ca}$ and $\mathrm{Pb}-\mathrm{Sm}$ titanates. J. Phys. D Appl. Phys., 1998, 31(4): 446.

[34] BOURIM E M, TANAKA H, GABBAY M, et al. Domain wall motion effect on the anelastic behavior in lead zirconate titanate piezoelectric ceramics. J. Appl. Phys., 2002, 91(10): 6662-6669.

[35] CHEN Y, WANG X S, LI Y S, et al. The low frequency relaxor properties of ferroelectric PZT-4 studied by DMA. J. Mater. Sci.
Mater. Electron., 2019, 30(8): 1-9.

[36] CHEN Y, YE H H, WANG X S, et al. Grain size effects on the electric and mechanical properties of submicro $\mathrm{BaTiO}_{3}$ ceramics. $J$. Euro. Ceram., 2020, 40(2): 391-400.

[37] KUMAR N, TIRUPATHI P, KUMAR B, et al. Observation of dielectric relaxor behavior in $\mathrm{Pb}_{0.95} \mathrm{Sr}_{0.05}\left(\mathrm{Zr}_{0.5} \mathrm{Ti}_{0.5}\right) \mathrm{O}_{3}$ ceramics. $A d v$. Mater. Lett., 2015, 64: 284-289.

[38] DA SILVA JR P S, VENET M, FLORÉNCIO O. Influence of diffuse phase transition on the anelastic behavior of Nb-doped $\mathrm{Pb}\left(\mathrm{Zr}_{0.53} \mathrm{Ti}_{0.47}\right) \mathrm{O}_{3}$ ceramics. J. Alloy. Comp., 2015, 647: 784-789.

[39] MAZUERA A, SILVA JR P, RODRIGUES A, et al. Origin of discrepancy between electrical and mechanical anomalies in lead-free (K, Na) $\mathrm{NbO}_{3}$-based ceramics. Phys. Rev. B, 2016, 94(18): 184101.

[40] CORDERO F, CRACIUN F, VERARDI P. Dielectric and anelastic relaxation in PMN-PT relaxors. Ferroelectrics, 2003, 290(1): 141-149.

[41] YU Y, WANG X S, LI Y S, et al. Fatigue behaviors in PZT ceramics induced by mechanical cyclic load. Ferroelectrics Lett., 2014, 41(4/5/6): 123-128.

[42] XIE S, XU J, CHEN Y, et al. Flexural fracture mechanisms and fatigue behaviors of $\mathrm{Bi}_{4} \mathrm{Ti}_{3} \mathrm{O}_{12}$-based high-temperature piezoceramics sintered at different temperatures. Ceram. Int., 2018, 44(14): 16758-16765.

[43] XIE S, XU J, CHEN Y, et al. Poling effect and sintering temperature dependence on fracture strength and fatigue properties of bismuth titanate based piezoceramics. Ceram. Int., 2018, 44(16): 20432-20440.

[44] ASMATULU R, CLAUS R, MECHAM J. Improving the damping properties of composites using ferroelectric inclusions. J. Intel. Mater. Syst. Str., 2005, 16(5): 463-468.

[45] SUMITA M, GOHDA H, ASAI S, et al. New damping materials composed of piezoelectric and electro-conductive, particle-filled polymer composites: effect of the electromechanical coupling factor. Makromol. Chem., Rapid Commun., 1991, 12(12): 657-661.

[46] YU J, KANEKO H, ASAI S, et al. Electrical and dynamic mechanical behavior of $\mathrm{BaTiO}_{3} / \mathrm{VGCF} / \mathrm{LDPE}$ composite. Compos. Interface., 2000, 7(5/6): 411-424.

[47] MARRA S, RAMESH K, DOUGLAS A. The mechanical properties of lead-titanate/polymer 0-3 composites. Compos. Sci. Techno., 1999, 59(14): 2163-2173.

[48] ZHANG C, HU Z, GAO G, et al. Damping behavior and acoustic performance of polyurethane/lead zirconate titanate ceramic composites. Mater. Design, 2013, 46: 503-510.

[49] HONG X Q, WANG X S, LI X M, et al. Damping properties of epoxy-embedded piezoelectric composites. Key Engineering Materials, 2012, 512-515: 1342-1346.

[50] SHI M X, HUANG Z X, WEI T, et al. Damping properties and mechanism of 0-3 PMN/CB/EP composites. Adv. Mater. Res., 2009, 66: $45-48$.

[51] 武传贵. 有机无机复合材料阻尼性能研究. 上海: 同济大学硕 士学位论文, 2011. 